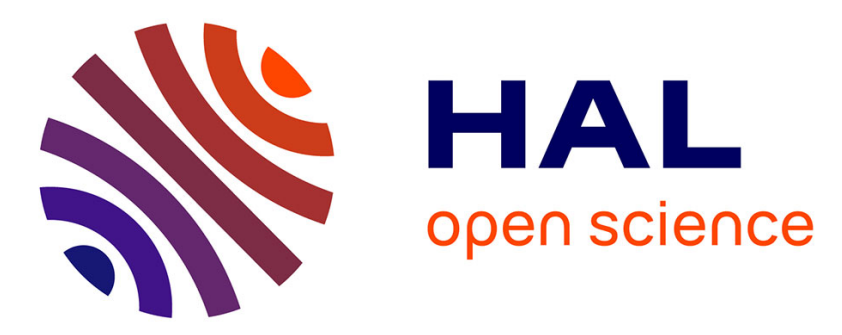

\title{
Amélioration de l'acquisition " en ligne " par calculateur CAE510
}

\author{
B. Cauvin, M. Huer, M. Sanche
}

\section{To cite this version:}

B. Cauvin, M. Huer, M. Sanche. Amélioration de l'acquisition " en ligne " par calculateur CAE510. Revue de Physique Appliquée, 1969, 4 (2), pp.124-126. 10.1051/rphysap:0196900402012401 . jpa00243175

\section{HAL Id: jpa-00243175 https://hal.science/jpa-00243175}

Submitted on 1 Jan 1969

HAL is a multi-disciplinary open access archive for the deposit and dissemination of scientific research documents, whether they are published or not. The documents may come from teaching and research institutions in France or abroad, or from public or private research centers.
L'archive ouverte pluridisciplinaire HAL, est destinée au dépôt et à la diffusion de documents scientifiques de niveau recherche, publiés ou non, émanant des établissements d'enseignement et de recherche français ou étrangers, des laboratoires publics ou privés. 


\title{
AMÉLIORATION DE L'AGQUISITION « EN LIGNE » PAR GALGULATEUR GAE 510
}

\author{
B. GAUVIN, M. HUET et M. SANCHE, \\ S.M.N.F., Centre d’Études Nucléaires de Saclay.
}

\begin{abstract}
Résumé. - Étude de l'amélioration par programme d'un système d'acquisition d'informations issues d'expériences neutroniques. Ce programme permet, sur un calculateur CAE 510 ne possédant pas l'accès direct en mémoire, d'exécuter un programme de fond interrompu périodiquement par l'acquisition des informations.
\end{abstract}

Abstract. - Improvements of a programme for data acquisition of information coming from neutron time of flight experiments are discussed. This programme, written for the CAE 510 computer (this computer does not permit direct access to the memory), is designed to permit execution of the main programme with periodic interruption for data acquisition.

I. Introduction. - Le calculateur est installé auprès de l'accélérateur $45 \mathrm{MeV}$ de Saclay et utilisé pour les expériences de temps-de-vol de neutrons. Six bases de temps-de-vol sont employées par les physiciens pour des expériences simultanées qui sont toutes susceptibles d'être traitées par des processus divers sur le calculateur. La mise en ligne des expériences a été exposée dans un article précédent [1]. Ce calculateur, type CAE 510, ne possède pas de fonction accès direct telle qu'elle existe dans la nouvelle génération de calculateurs. Seul un dispositif additionnel câblé (CAE 592) permet une entrée rapide $(30 \mu \mathrm{s})$ en mémoire centrale. Ce dispositif immobilise certains registres de l'unité de calcul, imposant des restrictions considérables à l'écriture d'un programme de fond : ce dernier ne peut donc être que rudimentaire (réalisant seulement un traitement élémentaire analogue à l'incrémentation des sélecteurs classiques). Pour une acquisition d'informations à faible taux de comptage (généralement inférieur à $3000 \mathrm{c} / \mathrm{s}$ ), l'unité centrale du calculateur n'est occupée que très partiellement. Le problème est d'utiliser le reste du temps pour l'exécution de programmes de fond plus élaborés.
II. Conception. - Le calculateur CAE 510 possède un dispositif, dit « d'interruption », permettant d'arrêter l'exécution d'un programme de fond pour entamer celle d'un programme prioritaire. La fin de l'exécution de ce programme prioritaire redonne le contrôle au programme de fond. Dans ce système, chaque programme dispose de la totalité des registres de l'unité de calcul, mais le passage de l'exécution de l'un à celle de l'autre nécessite des opérations longues (sauvegarde de registres, etc.).

Si l'on définit comme programme prioritaire l'acquisition des informations, il est clair qu'il faut éviter de faire intervenir le jeu de l'interruption pour chaque information, mais plutôt pour un groupe d'informations. Ces informations, au nombre de NINF, seront stockées dans une mémoire tampon avant d'être acceptées par le calculateur. Le temps d'attente sera utilisé pour l'exécution du programme de fond. Lorsque le nombre NINF informations est atteint, une échelle placée en parallèle sur la mémoire tampon envoie l'interruption IT au calculateur qui était dans la phase d'exécution du programme de fond.

Le programme d'acquisition prioritaire regroupe 


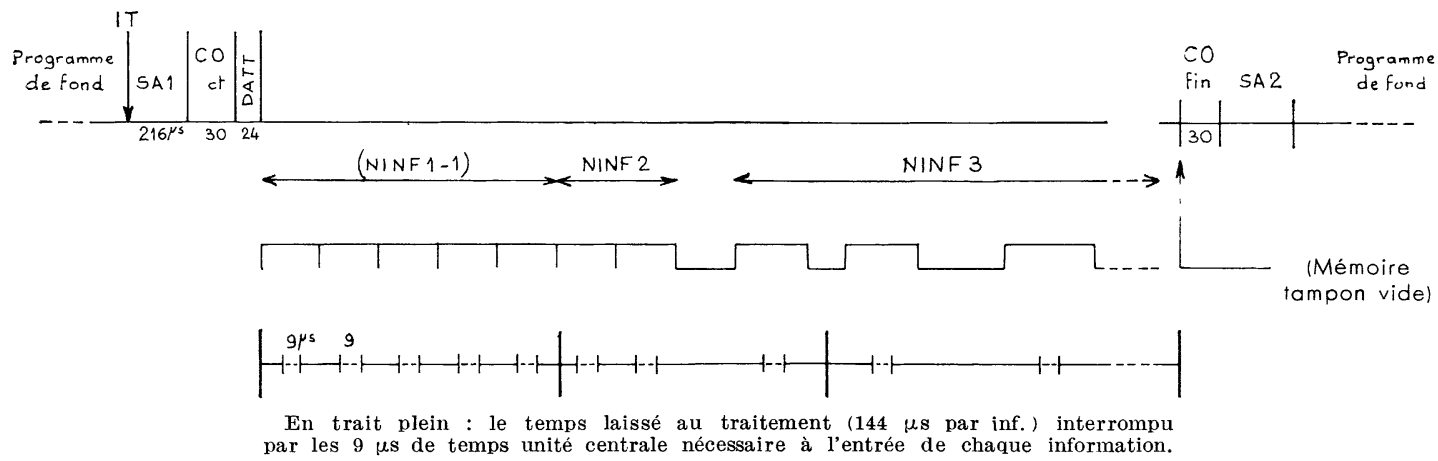

FIG. 1. - Diagramme séquentiel de l'acquisition en mode groupé : SA1, sauvegarde des registres ; CO act, passage au programme d'acquisition par activation CAE 592 ; DATT, attente pour entrée $1^{\text {re }}$ information; NINF1, nombre d'informations ayant déclenché l'IT; NINF2, nombre d'informations arrivées pendant SA1 et NINF1 * $33 \mu \mathrm{s} ;$ NINF3, nombre d'informations arrivées pendant le traitement; CO fin, fin du programme d'acquisition par désactivation CAE 592 ; SA2, restauration des registres.

Erratum : En haut à gauche, sous $\mathrm{CO}$, au lieu de ct, lire act.

l'acquisition proprement dite et le traitement élémentaire (incrémentation) primitif. La fin de l'exécution de ce programme est déclenchée par la détection de l'absence d'informations dans la mémoire tampon : on a ainsi acquis non seulement les NINF informations présentes à l'interruption, mais encore toutes celles qui sont arrivées pendant l'exécution du programme prioritaire. Le diagramme séquentiel est donné par la figure 1 .

III. Etude préliminaire. - Avant la réalisation pratique de ce système, une étude simulée a été faite par programme Fortran passé sur gros calculateur. Nous avons simulé l'arrivée des informations aléatoires par une méthode Monte-Carlo. Le programme gère l'histoire de chaque information dans une structure programmée en correspondance avec la structure câblée envisagée dans ce système. Un résultat important de celui-ci est le nombre d'informations perdues : la capacité de la mémoire tampon est limitée (11 adresses) et les pertes résultent principalement du dépassement de ces 11 adresses par les informations arrivant pendant le temps de sauvegarde des registres (SA1, voir fig. 1). Le taux de pertes dépend essentiellement du nombre prédéterminé NINF et du taux de comptage. Un autre résultat intéressant est le temps laissé par le programme prioritaire d'acquisition pour l'exécution du programme de fond.

Ces deux résultats sont donnés par la figure 2 . En abscisse, on a porté le taux de comptage; en ordonnée logarithmique, les valeurs du taux de pertes : $T=$ nombre de coups perdus/nombre de coups émis. En ordonnée linéaire sont portées les valeurs du pourcentage de temps utile du programme de fond : $P=$ temps progr. fond/temps total.

Le résultat montre que pour certaines valeurs du

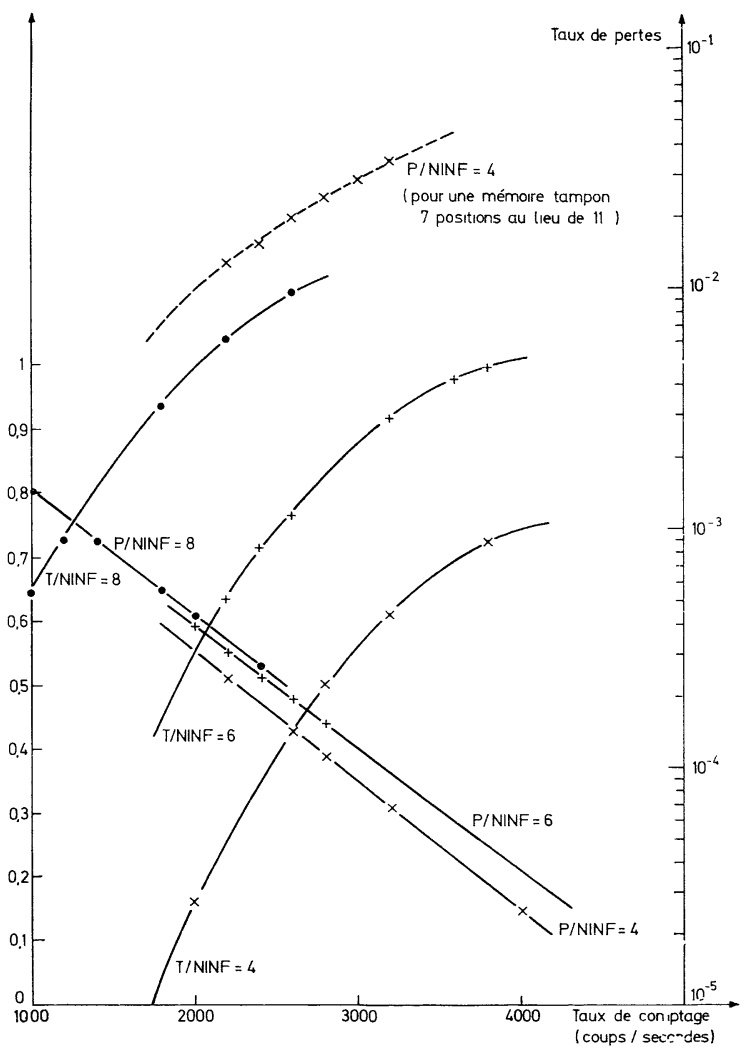

Fig. 2. - Taux de pertes et fraction de temps disponible pour le programme de fond :

En ordonnée, à gauche : $P$, fraction de temps disponible pour programme de fond; à droite : $T$, taux de pertes.

Erratum : En haut de la figure, sous la courbe en pointillé, au lieu de $\mathrm{P} / \mathrm{NINF}=4$, lire $\mathrm{T} / \mathrm{NINF}=4$. 
paramètre NINF et pour un taux de comptage moyen (environ $2000 \mathrm{c} / \mathrm{s}$ ), le taux de pertes est inférieur à $10^{-4}$ et le temps utile pour le programme de fond est de l'ordre de $55 \%$ du temps total. Ces résultats montrent qu'un tel système est rentable. La réalisation qui a suivi a donné des résultats en accord avec l'étude théorique.

IV. Utilisation en ligne du calculateur. - Ge programme d'acquisition est utilisé depuis un mois. Les programmes de fond choisis actuellement permettent essentiellement la matérialisation des résultats d'expérience par $: i)$ tracé de résultats antérieurs sur traceur Calcomp; ii) décodage et visualisation de blocs mémoires déconnectés de l'expérience en ligne; iii) visualisation simultanée de la zone d'acquisition en mémoire.

Le tracé se prête à cet exemple de multiprogrammation, l'unité centrale n'étant occupée que très partiellement.

Le temps d'utilisation du calculateur se répartit ainsi en plusieurs périodes dans la journée : 1) une période « en ligne » de traitement des bandes magnétiques 16 pistes emplies pendant la nuit précédente, suivie de l'exécution d'un programme « hors ligne » de dépouillement des informations prétriées (environ $5 \mathrm{~h})$; 2) une période "en ligne " d'acquisition des expériences qui s'étend sur toute la nuit. Cette période comporte simultanément les opérations possibles en programme de fond; 3 ) une période « hors ligne » de prédépouillement des expériences accumulées pendant la nuit (environ $2 \mathrm{~h}$ ). Le prédépouillement sur CAE 510, dont chaque partie peut se référer à tout moment au contrôle de la visualisation sur écran cathodique ou du tracé Calcomp, permet au physicien d'opérer lui-même et de maîtriser la première étape de condensation des résultats. Ces résultats, inscrits sur une bande magnétique directement relisible par les gros calculateurs du Département de Calcul Électronique, pourront ensuite être traités par un processus un peu plus automatique.

\section{BIBLIOGRAPHIE}

[1] Cauvin (B.), Ribon (P.) et Sanche (M.), Bull. Inf. Sci. Techn. C.E.A., 1967, 120. 